This document is published in:

Proceedings of Third International Conference on Digital Information Management. ICDIM 2008. IEEE, 2008, pp. 739-744. DOI: 10.1109/ICDIM.2008.4746697

(C) 2008 IEEE. Personal use of this material is permitted. Permission from IEEE must be obtained for all other uses, in any current or future media, including reprinting/republishing this material for advertising or promotional purposes, creating new collective works, for resale or redistribution to servers or lists, or reuse of any copyrighted component of this work in other works. 


\title{
MAW: A New Effective and Efficient Knowledge Meta-model for Audiovisual Works
}

\author{
Ana Iglesias, Guillermo G. Millán, Elisa Pintado, Elena Castro \\ Universidad Carlos III de Madrid \\ Avda. Universidad, 30. 28911 Leganés (Madrid) SPAIN \\ \{aiglesia@inf; epintado@di; ggmillan@di; ecastro@inf\}.uc3m.es
}

\begin{abstract}
To define an effective and efficient knowledge structure about audiovisual contents is not an easy task. Several research projects try to standardize the audiovisual data store and broadcasting process, as ISAN, VISAN, the EBU projects as ESCORT, TVAnytime or P/META, the SMEF meta-model from the $B B C$, etc. but all of them have a problem: they are defined in a specific domain, they are not generic, and they have not taken into account the importance of an effective and efficient definition of data. In this paper we propose a new and generic way for defining audiovisual works using the EntityRelationship model: MAW (Meta-model for Audiovisual Works). Moreover, we compare our solution with two of the most used meta-models: ISAN and SMEF
\end{abstract}

\section{Introduction and motivation}

Only few years ago, audiovisual works were distinguished one from others by their title and when a television broadcaster, for instance, wanted to broadcast a specific audiovisual material, they had to search it in their tapes. This process was completely inefficient and they wasted too much time finding the correct audiovisual work. Furthermore, they realized that the title of the audiovisual work was not enough to identify it. That is why the television broadcasters and television production houses firstly, and then the complete audiovisual sector realized the importance of identifying the audiovisual works and the need to define every characteristics of the work and store this information in an accessible and efficient system in order to search, retrieve, broadcast, publish, distribute, etc. these works in a fast way.

Different standards and initiatives to define audiovisual works are currently available, as ISAN and VISAN [8], the EBU projects as ESCORT [5], TV-Anytime [9] or P/META [6], the SMEF metamodel [1] from the BBC, etc., but each one has its own application field and features. Moreover, none of them is generic and most of them do not allow storing the data in an efficient way. For instance, data about captioning or audio description in audiovisual works are difficult to find in most of these standards or even, in some standards, this information is impossible to be retrieved. In addition, most of the standards mix different characteristics of the audiovisual works: definition characteristics, technical characteristics, production characteristics, broadcast characteristics, etc. and often repeated some information.

In this paper, we propose to use new metadata for audiovisual works with desirable characteristics: general metadata (non domain-specific), effective and efficient definition of the data avoiding data repetitions and distinguishing the different characteristics of the audiovisual works. This metadata, defined using the Entity-Relationship model (EER) [4][10], allows to store, search and retrieve information about audiovisual works in an efficient way. We have named this metadata model as MAW: Meta-model for Audiovisual Works. Moreover, in section 3 we present the advantages of MAW over two of the most used metadata for audiovisual works (ISAN and SMEF).

\section{Our proposal: Meta-model for audiovisual works (MAW)}

In this section, we present the new metadata proposed, where concepts that are related with audiovisual works and with their basic characteristics are represented. The meta-model is going to be described in different subsections, explaining the EER sub-schema of each section and the semantics assumptions needed to avoid the semantic lost [7],[3].

MAW proposal is a metadata representation built using the EER Conceptual Model and the [4] and [10] notation, so some assumptions must be considered for the implementation in a suitable database management system. The main objectives covered by MAW are: 
1. To differentiate whether the audiovisual work is an original one or a version.

2. To be able to classify the audiovisual works into serial collections (such as cartoon series or documentary series) when it will be appropriated.

3. To differentiate items or fragments of an audiovisual work (such as the scenes in a movie or parts of one movie appearing in other audiovisual work).

\subsection{Audiovisual work}

All audiovisual works (AW) are separated in two different types, original or version. An original work is the first work that is created. When an important change is made in the work, a new version appears. All versions are associated to an original work.

An audiovisual work is represented as a total and disjoint hierarchy with the AUDIOVISUAL WORK (AW) entity as the supertype and ORIGINAL and VERSION entities as subtypes. Main attributes are stored in AW in order to avoid repeated information and generate redundancy. Moreover, there is an attribute in VERSION called 'reason_version' that is useful to store the reason for creating the version. Figure 2 shows the described design.

Examples of semantic not included in the EER Schema is, for instance, that the AW attributes are mandatory for ORIGINAL audiovisual works but these attributes are optional for VERSIONS. In this case only modified fields from the original AW will be filled or the domain values of the attributes 'STATUS' or 'TYPE'.

\subsection{Media object group and media object}

An audiovisual work is composed of several elements or items. An element of an audiovisual work, for instance, is a mounted video with all his media components like images, audio, data, etc. An example could be the scenes of a movie.

The entity MEDIA OBJECT (MO) represents a media unit. Examples of media units are audio, video or data. When these units are grouped, a media object group will be created. In Figure 1, this relationship between MO and MEDIA_OBJECT_GROUPs (MOG) is represented. These groups are the elements or items of a full audiovisual work and are represented by the MOG entity.

In the EER schema proposed in Figure 2, MO is the super-type of a hierarchy formed by three subtypes: DATA, VIDEO and AUDIO

- DATA provides text, subtitles and all the content that can be displayed in an audiovisual work. The attribute 'FORMAT' will store if the format is txt, html, etc; and 'TYPE' will store the type of content.
- VIDEO provides all video items with no sound and with an attribute called 'GRAPHIC' to know if the image is real or synthetic.

- AUDIO stores all media sounds and has an attribute called 'TYPE' to know if it is an audio description, voice, or others.

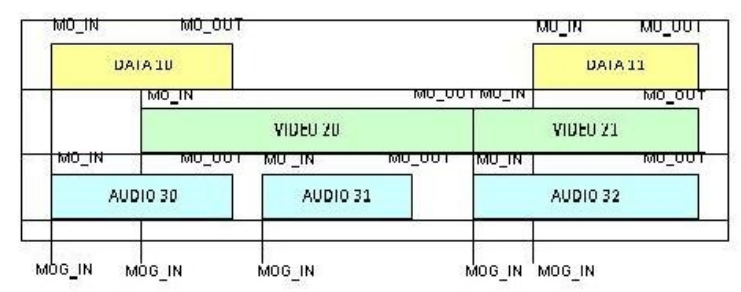

Figure 1. Timeline of a MEDIA_OBJECT_GROUP

Between MOG and MO there is a relationship called 'Composed by' which has three attributes, MO IN and MO OUT that are time marks used to select a part of a $\overline{M O}$, and MOG_IN that is the initial time mark that will tell us where to put the selected media object in the MOG.

To know which item or media object group is the original of an audiovisual work, an exclusive relationship exists between AW and MOG. The relationship 'Has' will store the original media object groups of an audiovisual work, while the other called 'Use' will store in which audiovisual works is the media object group used. It is represented by an exclusive relationship between $\mathrm{MOG}$ and $\mathrm{AW}$ because we want to know the original work that represent a MOG and differentiate it from other works, which may be composed of that same MOG. The relationship 'Has' in which it moves toward the key from AW to MOG, is for the parts that belong to a work, and a part or item can only be part of an original work. We will know for what audiovisual work was that part created. The relationship 'Use' tell us in which other works also appears that part or media object group, but those audiovisual works are not the original work. Therefore this exclusive relationship implements this type of association, in which one MOG belongs to an AW, but a MOG also can be part of other AW.

Examples of semantic not included in the EER Schema are, for instance, the domain values of the attributes 'GRAPHIC', 'FORMAT', 'COLOUR' or 'TYPE' or that the value of the attribute 'MO_IN' must be always previous to 'MO_OUT'.

Calculated Attributes:

(1) 'DURATION' in the entity MOG is calculated by adding the different durations of $\mathrm{MO}$ parts that compose it.

The duration of part of a MO for a MOG is:

MO.duration_for MOG $=$ MO_OUT $-\mathrm{MO}$ IN

The total duration of a MOG includes all parts of the MO that compose it. MOG.DURATION= ¿MO.DURATION_FOR_MOG

(2) 'DURATION' ' attribute in the entity AUDIOVISUAL_WORK is calculated by adding the times of each of the parts that compose the fund. 
Table 1. Calculated attributes

\begin{tabular}{|l|l|}
\hline$(1)$ & $\begin{array}{l}\text { MOG.DURATION }= \\
\sum \text { MO.DURATION_FOR_MOG }\end{array}$ \\
\hline$(2)$ & AW.DURATION $=\sum$ MOG.DURATION \\
\hline
\end{tabular}

\subsection{Audiovisual groups}

The AUDIOVISUAL_GROUP (AG) entity represents any kind of audiovisual works group of. In this proposal we differentiate two kind of groups, one for serials (documentary serials, cartoon serials, etc.) and the other for other kind of groups. It is represented in Figure 2 with a total and disjoint hierarchy having AG as supertype and SERIAL and GROUP like subtypes.

On the AW entity there is an attribute called 'TYPE' that gives information about the type of work that is going to be stored. For example, AW can be an episode of a series or a movie that is part of a compilation, among other types available.

The relationship 'Use' between AG and AW stores all the audiovisual works that are part of a group, and it has two attributes, 'ORDER_IN_GROUP' to know the order of the audiovisual work in the group, and 'SEASON' as an optional attribute to know the season that the work belongs to. An example is the 14th episode of the 6th season for a cartoon serial.

An example of semantic not included in the EER Schema is, for instance, that it is not possible to have the same season and order_in_group in the same AUDIOVISUAL_GROUP for different AUDIOVISUAL_WORKS. Moreover, these values should be ordered and consecutive numbers. Other example of semantic not included is that every AW that takes part of an AG.SERIAL must have the same type (AW.TYPE = 'episode').

\subsection{Language}

To store the different titles of any AG depending on the language, a relationship 'Has' is created.

For an AW, we need to store its title and the languages in which that audiovisual work is dubbed. We consider that an audiovisual work can be dubbed into more than one language and every AW will always have a title because the original language of a work will define it, even if it is a silent work.

Moreover, the 'Spoken' relationship stores information about other languages that are spoken in the AW (for instance, an English movie where Spanish words are incorporated) and the 'Has' relationship stores common information for audiovisual groups in order to avoid data redundancy.

\subsection{Participants}

Every human participant in an AW or SERIAL will take part into the 'WorksAW' or 'WorksSerial' relationships.

The 'WorksSerial' relationship stores information about the actor, directors, etc. In a serial group, for avoiding data redundancy, if this data change in an episode of this serial, the 'WorksAW' relationship will store this information (see Figure 2).

The attribute called 'TYPE' will define the type of participation of the human person in the AW or SERIAL. It can be actor, director, productor or others. TYPE $=$ AACTOR, DIRECTOR, PRODUCTOR, CAMERAMAN, OTHERS $\}$.

\subsection{Country and production companies}

The country of an audiovisual group is stored in the relationship 'Produces in' between SERIAL and COUNTRY entities showed in Figure 2.

The production company of an audiovisual group is stored in the relationship 'Has' between SERIAL and PRODUCTION COMPANY entities. This relationships will serve for all the audiovisual works, if there is any change in one audiovisual work, it will be stored in the respective relationships 'Produces in' and 'Has' with AW.

An example of semantic not included in the EER Schema is that in the hierarchy of AG which has two subtypes, SERIAL and GROUP, in subtype SERIAL are stored data from participants with the relationship 'Works' to the entity PARTICIPANTS, data from the production company with the relationship 'Has' to the entity PRODUCTION_COMPANY and the data of the country with the relationship 'Produces in' to the entity COUNTRY. These relationships that exist in the entity AG.SERIAL also occur with AW, but for $\mathrm{AW}$ that are associated to an $\mathrm{AG}$; these $\mathrm{AW}$ will only be filled if there is any modification for the AW.

\subsection{Rate}

This entity will store the rating and the content alerts of an audiovisual work. Diferent countries have diferent rating systems, and one rating will have one or more content alerts, that is the reason of the creation of an entity RATE and a multivaluated attribute called 'CONTENT_ALERTS'. 


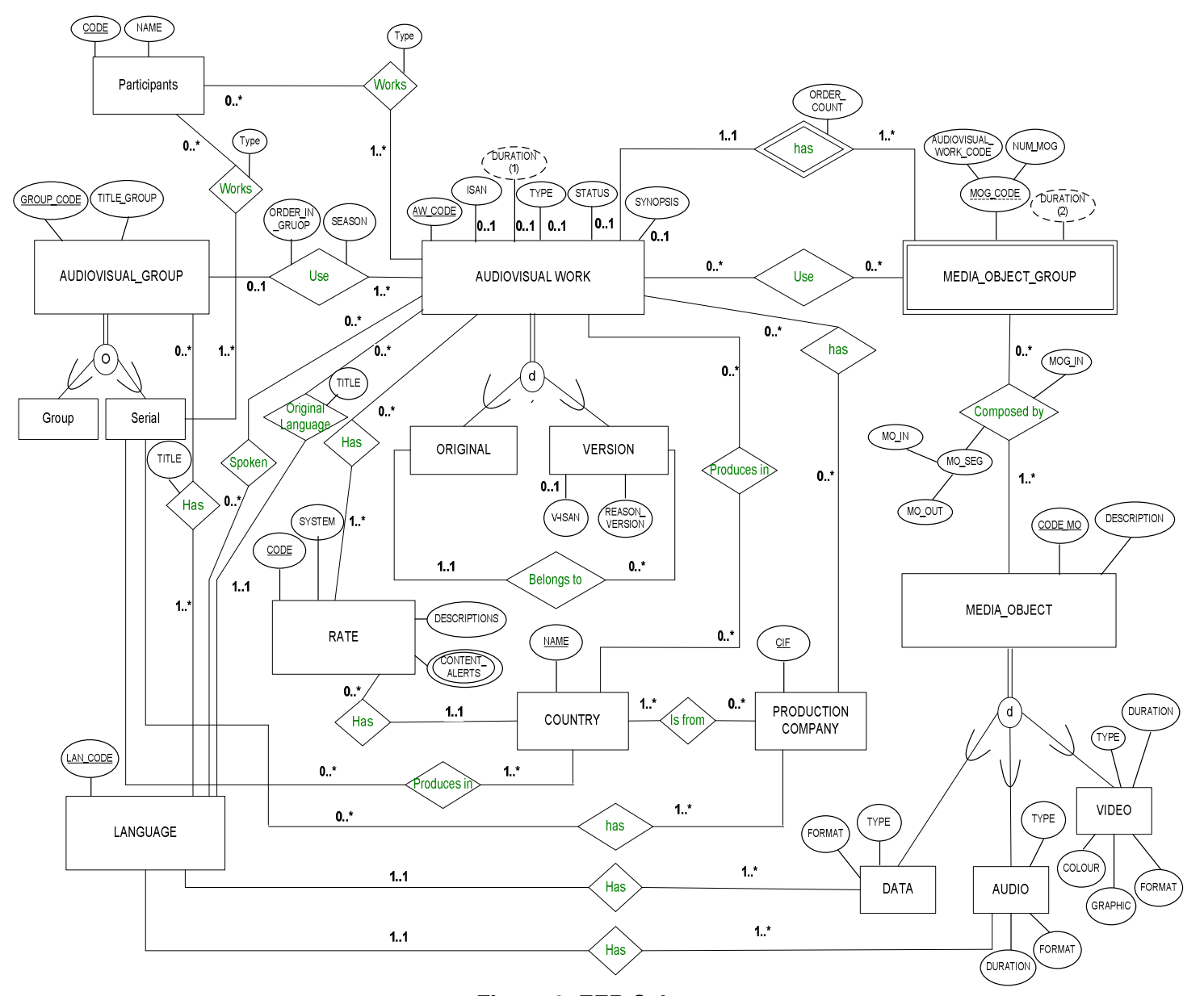

Figure 2. EER Schema

\section{Advantages of MAW over ISAN and SMEF metadata}

The way in which ISAN stores audiovisual works is very useful in terms of the overall vision that has of them. This standard is much clearer than the SMEF standard because it explains every entity and relationship, but ISAN standard stores less data about audiovisual works than SMEF. On the other hand, SMEF provides a glimpse of audiovisual works in detail, but it allows much more data redundancy. With the knowledge gained by studying these two standards this proposal has being achieved, which includes the good parts of each one, along with new proposals that simplify the storage of audiovisual works, representing an improvement for defining them.

Many ideas of SMEF had been taken into account for MAW, using too some ideas of ISAN and including new concepts and ideas. Next a comparative of the three models is described.

\subsection{Originals and versions of audiovisual} works
ISAN standard distinguishes between original works and versions of the audiovisual works, representing them as different entities. Moreover, a relationship exists between the two entities which indicate that an original work may have none or several versions associated, and at a same time, a version only belongs to one original work. In ISAN standard it is not mandatory to have a version of an audiovisual work. ISAN separates the idea of original works and versions. Moreover, using the entity VERSION, ISAN stores the changes of this version in a descriptive mode. It has some attributes as the length, language, etc. that allows to store some differences in an efficient way, but it has other attributes as 'description' that allows to describe in text the main differences between the original version and this version. These kind of attributes are not efficient for retrieving information.

On the other hand, SMEF standard, instead of dividing these concepts (ORIGINAL and VERSION), proposes a different idea. There is an abstract entity in its metadata description that represents an audiovisual work without taking into account their different versions or releases in different countries. This entity is known as the concept of an audiovisual work and this entity is called EDITORIAL_OBJECT_CONCEPT (EOC). 
In SMEF standard, one EOC is associated with one or more EDITORIAL_OBJECT_VERSION (EOV). This entity collects all information relating to a specific version of an audiovisual work, being the original version of the work or another version. All relationships and attributes that an audiovisual work can store will be associated with this entity, because EOC simply defines the concept of an audiovisual work, saving its ISAN code and the name of the concept, in a way that serves to make groups of related versions. This makes a lot of relationships with the entity EOV, besides a clear relationship between an original audiovisual work and its version is not saved. Moreover, in SMEF a version can be a publishable program or an item that cannot be published itself, but rather form a part of a program that is publishable. Furthermore, SMEF standard has an entity called EDITORIAL_OBJECT ASSOCIATION (EOA), which saves the associations between two versions of audiovisual works. This entity is used to store the association between a program and the items that comprise, in addition to containing the order in which they appear. SMEF also creates an entity to store the types of associations, EDITORIAL_OBJECT_ASSOCIA-TION_TYPE related with EOA. Finally, SMEF standard stores the version creation reason in the EDITORIAL_VERSION_CREATION_REASON TYPE entity, which relates each version with the reason for its creation, with a name and a description that gives us additional information to know the reasons of the creation. This information is descriptive, as in ISAN standard.

The advantages in our proposal are described next. A hierarchy has been created for audiovisual works whose subtypes can be original works and versions.

In ISAN this hierarchy doesn't exist, but distinguishes original works from versions and it associates them. The way ISAN does it is not effective nor efficient, because it does not allow recovering information in an easy way, for instance, the new actors in a extended version of a movie. This is solved in our proposal with the hierarchy making all the relationships with the AUDIOVISUAL WORK supertype and we avoid repeating data filling the VERSION attributes only when they change respect to the ORIGINAL version of the audiovisual work. Our proposal, as ISAN standard, also provides information about which version is related to which original work in an easy way. SMEF stores this information in a much more complicated way. It has the EOA entity (instead of a relationship) with two relationships with EDITORIAL_OBJECT VERSION.

On the other hand, in our proposal we consider a version as a complete audiovisual work, but in SMEF standard, versions can be parts of jobs that can't be publishable for themselves, called ITEM. We think that this information should be stored as items of audiovisual works.

\subsection{Serial and composition}

ISAN proposes a hierarchy in which distinguishes an audiovisual work being serial or not serial, so it distinguishes, for instance, if it is a film (not serial) or an episode of a series (serial). To group together all episodes of a same series ISAN uses the entity SERIAL HEADER, which stores the name of the serial and it stores too the language of the serial. In our proposal we allow to store common values of every episode of the serial group, but not only the serial title, but also their participants, production country, languages, etc. We avoid repeating all this information for each episode, avoiding data redundancy. Moreover, ISAN has a relationship of the entity AUDIOVISUAL WORK to itself to create audiovisual works that are composed of other complete audiovisual works. It does not give the possibility of creating an audiovisual work composed of parts from other works. Our proposal allows composing audiovisual works from other AW.

On the other hand, SMEF has an entity that is a group which is directly related to the concept and version. This entity (EDITORIAL OBJECT GROUP) serves to group common versions or to group audiovisual works in a specific series. It is useful to identify group of episodes that compose serial works and to detail the concept of the same. That is why we adopt part of this solution. In SMEF standard, a group can consist of other groups, but they will not be instantiated in this entity, to this end we use a relationship called EDITORIAL_OBJECT GROUP_IN_GROUP.

The advantages of our proposal are described next. A hierarchy has been created with a supertype called AUDIOVISUAL_GROUP, which distinguishes serial groups with other types of groups. In this way we can save common characteristics of the series as the title, languages, producer, actors, director, etc. and it is not necessary to repeat information in each episode that belongs to the same series. ISAN does not store this information in an efficient way, only keeps the header, the title of the series, but not the characteristics of the series.

Moreover, ISAN does not allow grouping parts of an audiovisual work, only the complete work. In our proposal this problem is resolved in the next section (3.3) with the entity MEDIA_OBJECT_GROUP.

Finally, SMEF makes many groupings, with the concepts, versions, media object group, instances, etc., and many groupings create redundancy in the data, which is why in our proposal we decided to group series and other groups in the hierarchy AUDIOVISUAL_GROUP. 


\subsection{Media object}

In ISAN standard this information is not stored. They store information about complete audiovisual work. The purpose of ISAN is to provide a unique identification number for complete audiovisual works.

On the other hand, in SMEF standard the MEDIA_OBJECT entity describes the audiovisual work components. It may represent for example, audio, video, subtitles or audio descriptions. Each MEDIA_OBJECT is a single type, one will be a video, and other will represent data, and so on. In addition there is another entity called MEDIA OBJECT GROUP that stores relationships with audiovisual concepts, or relationships of a version specifically with MEDIA OBJECT. Especially audio tracks and video are grouped together in the editing process for facilitating the production process. SMEF has MEDIA OBJECT GROUP on the one hand and on the other hand MEDIA_OBJECT. And depending on the use to which a version is designated, it relates to EDITORIAL OBJECT VERSION. Besides a version have subtypes like programs that are fully publishable, and parts or items of a program. It keeps the relationship between the groups of MEDIA_OBJECT with its concept or with the group to which it belongs.

The part related with MEDIA_OBJECT, which appears in SMEF seems to us a very interesting and essential concept when it comes to storing audiovisual objects, but we have made some changes. The hierarchy that has MO in our proposal consists of three subtypes: DATA, VIDEO and AUDIO, many subtypes like in SMEF have not been implemented. We have simplified this hierarchy, in only three types of basic data of a MO, with these three types we can contemplate the same possibilities as SMEF. In our proposal, the TIMELINE MEDIA OBJECT is not consider a MO like SMEF did, because it does not represent an audiovisual object but it is the chronological order in which appear MO, to do so in the relationship 'Composed by' between $\mathrm{MO}$ and MOG some attributes that represent what has been stated are stored, MOG IN to know in order to appear, MO IN and MO OUT to know the part of the MO we are going to take.

\section{Conclusions}

In this paper, we present a new proposal of metadata for storing in an effective and efficient way audiovisual works data in order to search, retrieve, etc. each AW characteristic. With this proposal we avoid the difficulties derived from data redundancy or access impossibility to the data because of the definition of the metadata model. The meta-model proposed in this paper is generic (is not defined for a specific domain) and the effective and efficiency data store and data recovery has been taken into account in every moment, trying not to mix different types of AW characteristics. For instance, we try not to mix technical characteristics with production characteristics. Moreover, this paper shows the main advantages of our proposal over ISAN and SMEF standards, two of the most used metadata standards for audiovisual works.

MAW is successfully been used in the CESyA ${ }^{1}$ database (SABADO) with information of audiovisual works with captioning and/or audiodescription.

\section{References}

[1] BBC Technology: SMEF data model version 1.10. Technical report, 2004. Available in http://www.bbc.co.uk/guidelines/smef/.

[2] Camps, R. From ternary relationship to relational tables: a case against common beliefs. ACM SIGMOD Record, v.31 n.2. pp. 46-49, 2002.

[3] Cuadra D., Nieto C., Martínez P., Castro E. and Velasco M. Database Integrity: Challenges and Solutions. Idea Group Publishing, 2002.

[4] Elmasri, R. and Navathe, S.B. Fundamentals of Databases Systems, 5th Ed. Pearson Addison-Wesley, 2006.

[5] European Broadcasting Union: Escort: EBU System of Classification of RTV Programmes. Technical report, 1995. Available in

http://www.ebu.ch/en/technical/metadata/specifications.

[6] European Broadcasting Union: P/Meta Metadata Exchange Scheme v1.1. Technical Report Tech. 3295 2005. Available

http://www.ebu.ch/en/technical/metadata/specifications/not es on tech3295.php.

[7] Gogolla, M. and Hohenstein, U. Towards a semantic view of an extended entity-relationship model, $A C M$ Transactions on Database Systems (TODS), v.16 n.3, p.369-416, 2004.

[8] ISAN: Information and documentation International Standard Audiovisual Number (ISAN). ISO 15706, 2002

[9] J.-P. Evain: TV-Anytime metadata: a preliminary specification on schedule! EBU Technical Review No.284, 2000.

[10] Silberschatz A., Korth H.F. and Sudarshan S. Database Systems Concepts. 5th Ed. McGraw-Hill Companies, 2005.

\footnotetext{
${ }^{1}$ CESyA is the Spanish Center of Captioning and Audiodescription (www.cesya.es)
} 